\title{
'Fragile' liver and massive hepatic haemorrhage due to hereditary amyloidosis
}

\author{
R F Harrison, P N Hawkins, W R Roche, R F T MacMahon, S G Hubscher, \\ J A C Buckels
}

\begin{abstract}
The first case of amyloidosis is reported in which spontaneous massive hepatic haemorrhage necessitated emergency liver transplantation. Liver transplantation, as a treatment for a failing liver due to amyloidosis has not been previously reported. At transplantation, the liver tissue was uncharacteristically friable, although the subsequent vascular and biliary anastomoses were uncomplicated. Histological examination of the liver showed a surprisingly modest amount of amyloid, which was shown immunohistochemically to be derived from lysozyme, and a striking absence of reticulin staining. Both the patient's father and paternal grandfather had died from spontaneous hepatic haemorrhage, and histological review of their liver tissue showed similarly modest deposition of lysozymederived amyloid associated with loss of reticulin staining. In each case the quantity of amyloid was far less than would be expected to interfere with the mechanical integrity of the liver. This is the only report of hepatic disintegration associated with absence of reticulin staining, and it is probable that the mechanism represents a novel secondary effect of the amyloid deposits in the livers of this family.

(Gut 1996; 38: 151-152)
\end{abstract}

Keywords: hereditary amyloidosis, liver, haemorrhage.

\section{Case report}

A 15 year old male presented with a two day history of worsening upper abdominal pain, referred to the shoulder tip. At the age of 13, a diagnosis of amyloidosis had been made on rectal biopsy. On admission he was in shock, and underwent emergency laparotomy. The operative findings were those of a large bleed into the abdominal cavity and a large subcapsular haematoma over the upper aspect of the whole liver. The clot was evacuated and the liver packed. He was transferred to a specialist hepatobiliary unit after 24 hours of intensive care, but soon required another laparotomy for evacuation of a further blood clot, packing, and spraying of the involved area with fibrin glue. Liver function deteriorated, however, necessitating emergency liver transplantation. At transplantation the liver was noted to be remarkably fragile such that it was 'falling apart'. At six months post-transplant the patient is well, and there have been no anastomotic problems.
The patient's father underwent investigation for multiple hepatic bleeds requiring laparotomy for evacuation of hepatic haematoma and repeated transfusions. No coagulation defect was found, but there was prolonged bleeding time, and liver and rectal biopsy specimens showed amyloidosis. Coeliac arteriography and hepatic scans showed filling defects and calcification in the liver. At the age of 35, in view of repeated bleeding, he underwent hepatic artery ligation, but intraoperative bleeding was uncontrollable and he died soon after.

At the age of 50 , the paternal grandfather died shortly after uncontrollable hepatic haemorrhage during portocaval anastomosis for portal hypertension. At operation there was a fistula between the portal vein and the common bile duct. There was a 10 year history of hepatic dysfunction, haematemesis, and portal hypertension.

\section{Pathological findings}

The patient's explanted liver weighed $1800 \mathrm{~g}$. It was remarkably friable with a crumbly texture and golden-yellow appearance; it resembled necrotic tissue. There was extensive haemorrhage into the parenchyma. Microscopically, portal tracts contained amorphous deposits of hyaline material (Fig 1), confirmed to be amyloid by Congo red staining. Minor amyloid deposits were seen in the space of Disse and in the walls of medium sized blood vessels (arteries and veins). Immunohistochemical studies were performed using a panel of antibodies against amyloid fibril proteins (AA amyloid, $\kappa$ and $\lambda$ light chains), which were negative. A specific positive reaction was obtained with rabbit antilysozyme antibody (Dako) showing that lysozyme was a major constituent of the amyloid. Staining for reticulin using the Gordon Sweets method

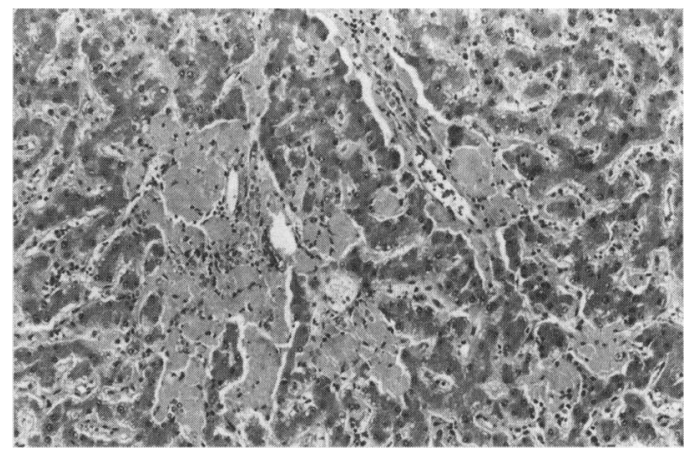

Figure 1: Portal tract containing globular deposits of amyloid; only modest amounts of amyloid are seen in the space of Disse (haematoxylin and eosin $\times 200$ ). 


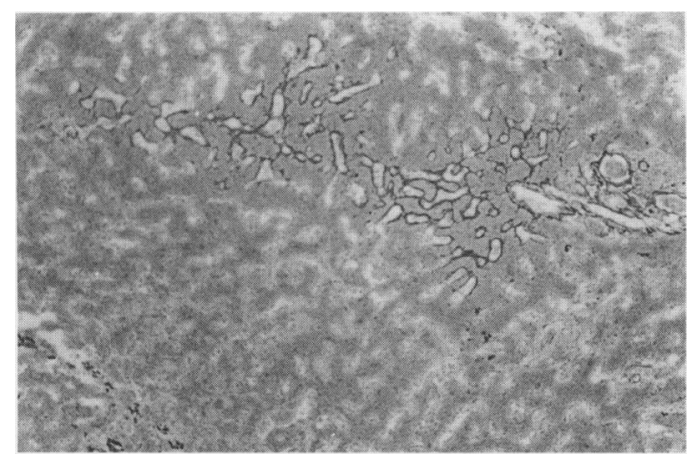

Figure 2: Large areas of liver are devoid of reticulin staining; only small patches around portal tracts remain (reticulin $\times 100$ ).

showed that the normal reticulin framework was absent in large areas of the liver. A few areas still retained residual reticulin fibres (Fig 2). Overlaying of slides showed that the presence of amyloid corresponded to the absence of reticulin staining. Immunohistochemical staining for collagen subtypes I, III, IV, and V showed that type IV collagen was still present beneath the amyloid deposits. Extensive centrilobular zonal necrosis was present reflecting ischaemic damage due to hypoperfusion. Amyloid was also seen in a portal lymph node, but not in a renal biopsy specimen taken during assessment of renal function around the time of transplantation.

At necropsy, the father's liver was large $(3420 \mathrm{~g})$ and contained fresh and partially organised haematomas; the parenchyma was a golden-brown colour. The spleen was also enlarged at $750 \mathrm{~g}$. Histological examination of the liver showed irregular fibrous scarring, presumably a reflection of previous bleeds. There were areas of haemorrhage and necrosis. Portal tracts, and to a lesser extent sinusoids, were infiltrated by lysozyme amyloid. There was loss of reticulin staining around liver cell plates. In other tissues, widespread deposition of amyloid was seen in the walls of blood vessels; globular deposits of amyloid were seen in lymph nodes but not in the kidneys or heart.

At necropsy, the grandfather's liver was described as 'pultaceous', large $(2430 \mathrm{~g})$, and diffusely yellow and necrotic in appearance. Review of the histological findings showed extensive centrilobular zonal necrosis and deposition of lysozyme amyloid in portal tracts. A pattern of reticulin loss similar to that seen in the son's and grandson's liver was seen; amyloid was also present in the spleen.

\section{Discussion}

We report the first case of massive hepatic haemorrhage due to hereditary systemic amyloidosis necessitating liver transplantation. Liver transplantation has previously only been used as a surgical form of 'gene therapy' in patients with familial amyloid polyneuropathy in whom the amyloid fibril precursor protein is exclusively produced in this organ. ${ }^{1}$ In our patient, liver transplantation is palliative as lysozyme is synthesised by macrophages throughout the body and it is therefore probable that hepatic amyloid deposition will recur in the transplanted organ. The identification of lysozyme as the amyloid fibril protein, together with the autosomal dominant inheritance, strongly suggest that a variant form of lysozyme, due to a mutation in the lysozyme gene is responsible for the disease in this family. DNA studies are in progress, and will be the subject of another report.

Only two families with hereditary lysozymeassociated amyloidosis have been described, and in neither was reticulin staining or hepatic haemorrhage a feature despite the fact that several of these patients had massive hepatic amyloid deposits. ${ }^{2}$ Haemorrhage is a recognised, though rare complication in hepatic amyloid, mainly following biopsy when the liver can fracture. ${ }^{3}$ Typically in such cases, the liver is waxy and rigid. ${ }^{4}$ In contrast, the striking feature of the liver in this report was its fragility and crumbly consistency. Clinical records point to a similar hepatic appearance in the two other patients in this family. In the liver, type IV collagen is a major component of 'reticulin' on which the liver cell plates rest, and it maintains sinusoidal integrity. ${ }^{5}$

Histologically the loss of reticulin staining coincided with amyloid deposition in the sinusoids, suggesting a direct relation between the two. It has been suggested that lysozyme can digest the reticulin framework of the liver ${ }^{6}$ but these findings could not be confirmed in more recent work ( $T$ Krausz and M B Pepys, personal communication). The maintenance of staining for type IV collagen implies that it is still present beneath the amyloid, but that it is not staining with the silver stain for reticulin. Nevertheless, these findings suggest an unusual mechanism for the hepatic bleeding, and we speculate that the loss of reticulin staining, a common factor among the three cases is associated with the liver fragility. It seems that amyloid deposition can, at least in some circumstances, exert important secondary effects on the mechanical integrity of the liver. We believe that this is the first report in which reticulin staining has disappeared from the liver.

1 Steen L, Holmgren G, Suhr O, Wikstrom L, Groth C-G, Ericson B-G. World-wide survey of liver transplantation in patients with familial amyoloidotic polyneuropathy. Amyloid 1994; 1: 138-42.

2 Pepys MB, Hawkins PN, Booth DR, Vigushin DM, Tennent GA, Soutar AK, et al. Human lysozyme gene mutations cause hereditary systemic amyloidosis. Nature 1993; 362: 553-7.

3 Schaffner F. Liver biopsy. In: MacSween RNM, Anthony PP, Scheuer PJ, eds. Pathology of the liver. 2nd ed. Edinburgh: Churchill Livingstone, 1986: 692 .

4 Levine AD. Amyloid disease of the liver. $A m \mathcal{F} M e d 1962 ; 33$ : 349-57.

5 Burt AD, Griffiths MR, Schuppan D, Voss B, MacSween RNM. Ultrastructural localisation of extracellular matrix proteins in liver biopsies using ultracryomicrotomy and immunogold labelling. Histopathology 1990; 16: 53-8.

6 Velican D, Velican C. Lysozyme and the pericapillary reticulin network. Nature 1967; 215: 889-90. 